\title{
Monitoring proteins and protein networks using reverse phase protein arrays
}

\author{
Bridget Wilson, Lance A. Liotta and Emanuel Petricoin III* \\ Center for Applied Proteomics and Molecular Medicine, George Mason University, Manassas, VA, USA
}

\begin{abstract}
Recent advances in high throughput, high content "omic" technologies coupled with clinical information has lead to the expectation that the complexity of the molecular information generated will lead to more robust scientific research as well as the expectation that overarching therapeutic approaches will be patient-tailored to the underlying specific molecular defects of the disease. As disease understanding progresses and more therapeutics, which predominately target proteins, are developed there is a need to more confidently determine the protein signaling events that can be correlated with drug response since the deranged protein signaling networks are often the drug target itself. In this environment, the Reverse Phase Protein Microarray (RPMA) can be utilized to address the needs of both clinical screening and disease understanding through its ability to provide an unmatched functional and highly multiplexed signaling network level mapping of ongoing signaling activation, coupled with the ability of the platform to provide this information reproducibly from a tiny needle biopsy specimen or fine needle aspirate. This platform has now been utilized for biomarker discovery/validation and advancements in disease understanding both in the clinic and at the bench in the fields of cancer, liver disease, immunological disorders, and bacterial infection.
\end{abstract}

\section{Introduction}

As individualized medicine pushes the limits of scientific exploration and understanding, the complexities involved in mapping human genetic profiles continues to create more questions than answers. Advancements in the understanding of disease have required contributions from both the fields of genomics and proteomics. The combination of these two fields paints a complete picture of disease progression and potential causes. Genomics is able to identify mutations, polymorphisms, and splice variants. Proteomics focuses on variations in signaling from cell to cell, signaling changes that occur over time, and the ultimate effect on protein function resulting from gene alterations. While recent findings in diseases such as cancer reveal mutational underpinnings, each patient displays a distinct mutational fingerprint and at a functional level, these mutations correspond to distinct pathway effects. Thus, protein me-

${ }^{*}$ Corresponding author: Emanuel Petricoin III, 10900 University Blvd, Manassas, VA 20155, USA. Tel.: +1 703993 8646; E-mail: epetrico@gmu.edu. diated signaling is both directly and indirectly effected by these mutational alterations in addition to influences of the tissue microenvironment. This has lead to a need to establish an understanding of protein function within the unique cellular microenvironment, and to show how protein pathways are linked and coordinately regulate cellular processes. These findings can ultimately be applied to disease understanding, advancements in diagnostics, specific drug targeting, and therapeutic response monitoring $[8,13,17,24,27]$.

Microarrays have arisen to be an effective and versatile platform from which to conduct research. Microarrays have been utilized to profile disease at the DNA, RNA, and protein levels. This has led to discoveries of disease susceptibility genes, gene alterations, drug targets, and protein expression profiles correlated to disease outcome and drug response. Despite the contributions made by molecular profiling, the effort has been unable to capture the complexity of the cellular protein networks for several reasons $[27,28]$. First, gene transcript levels have not been significantly correlated with protein expression levels. In addition, RNA transcript levels provide little insight into the state of cellular signaling networks or protein-protein interactions, making 


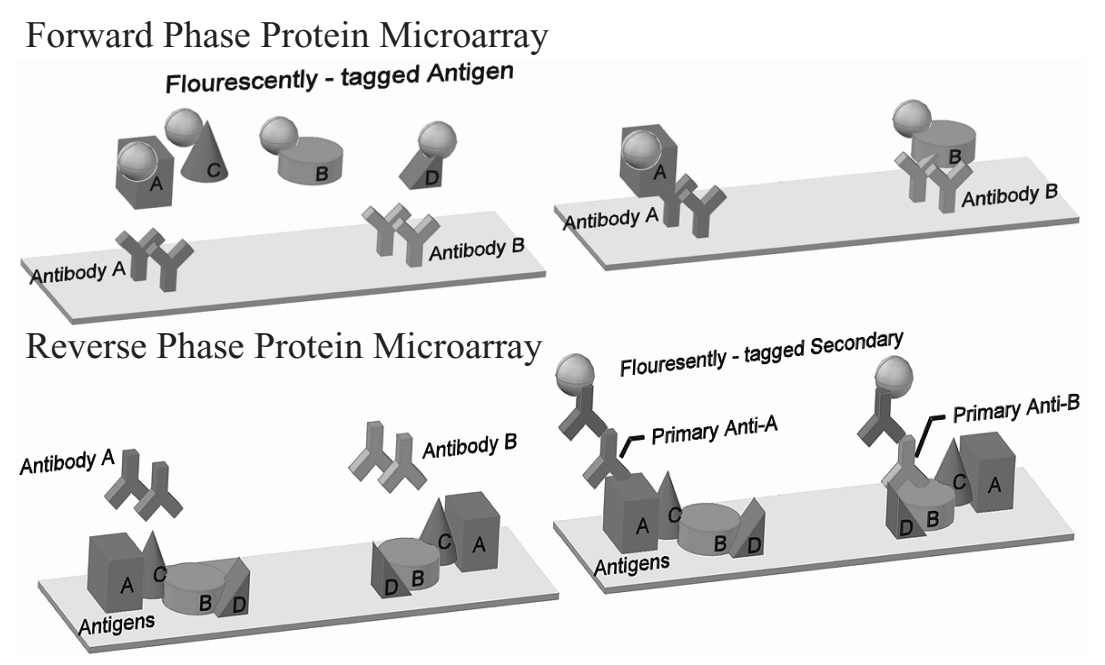

Fig. 1. Protein microarray platforms. Forward phase arrays (top) immobilize multiple antibodies that are individually spotted and designed to bind specific antigen within a sample. Bound antigens are then detected through direct labeling. Reverse phase arrays (bottom) immobilize multiple protein samples, also spotted individually. An antigen specific primary antibody binds the protein of interest, followed by a secondary antibody with a fluorescent tag.

it necessary to directly investigate protein-driven signaling. Lastly, most therapeutic agents target proteins not genes, and these proteins are most often protein kinases and/or their substrates. The activation state of these proteins and the signaling networks within which they are subsumed are in a constant state of change [27, 28]. This dynamic interplay is not observable within DNA and RNA based molecular studies. In order to generate information regarding these signaling network interactions, there is a need to utilize a proteomic-based platform.

Protein arrays fall into two general classifications: forward phase arrays and reverse phase arrays. Forward phase protein microarrays (FPPM) involve the immobilization of multiple antibodies, which are individually spotted on the array surface, followed by exposure to a single protein sample. In contrast, reverse phase protein microarrays (RPMA) entail the deposition of multiple protein samples, also individually spotted, and then probed with a single validated antibody (Fig. 1). RPMA, unlike other protein arrays, involves the immobilization of all proteins present in an individual tissue or cell population instead of a single probe. The RPMA enables use of a smaller sample size and enables all experimental samples to be processed under the same experimental conditions [10,13,16,27]. A further benefit of the RPMA platform is utilization of denatured lysates to enhance antigen retrieval or non-denatured lysates, such as microdissected tissue, to enable monitoring of protein interaction to include protein-protein, protein-DNA, and protein-RNA.
In general an RPMA experimental procedure requires samples to be deposited onto a nitrocellulose coated slide and arrayed in a serial dilution to provide accurate quantification through enlarging the dynamic range and to serve as an internal standard [16,22]. The array is then probed with an antibody shown to be specific for the target protein, which is usually determined via western blot. Next, the array is incubated with a biotinylated secondary antibody that binds to the primary antibody. Lastly, a streptavadin-linked label, either fluorophore or colormetric, binds to the biotin to enable visualization. The array is then imaged, analyzed, and interpreted. For each spot, the intensity value is proportional to the quantity of primary antibody bound to the protein of interest within each protein sample present [13,27].

There are a few pivotal strengths that the RPMA platform has over other protein detection systems. It is a well-known fact that proteins are unable to be amplified by a technique such as polymerase chain reaction; so, this creates a need for a method that gives a robust signal and only requires a small amount of sample. A conventional western blot requires $5 \times 10^{5}$ cells for adequate detection, whereas the RPMA requires only around 200 cells per array [23]. In addition, the RPMA platform exhibits extremely sensitive analyte detection with levels as low as $1.0 \times 10^{-21} \mathrm{~mol}$ or $1.0 \times 10^{-18}$ $\mathrm{g}$ for a given protein and an ability to detect a variance of less then $10 \%$. Sensitivity is achieved through the ability to easily obtain the linear range through spotting 


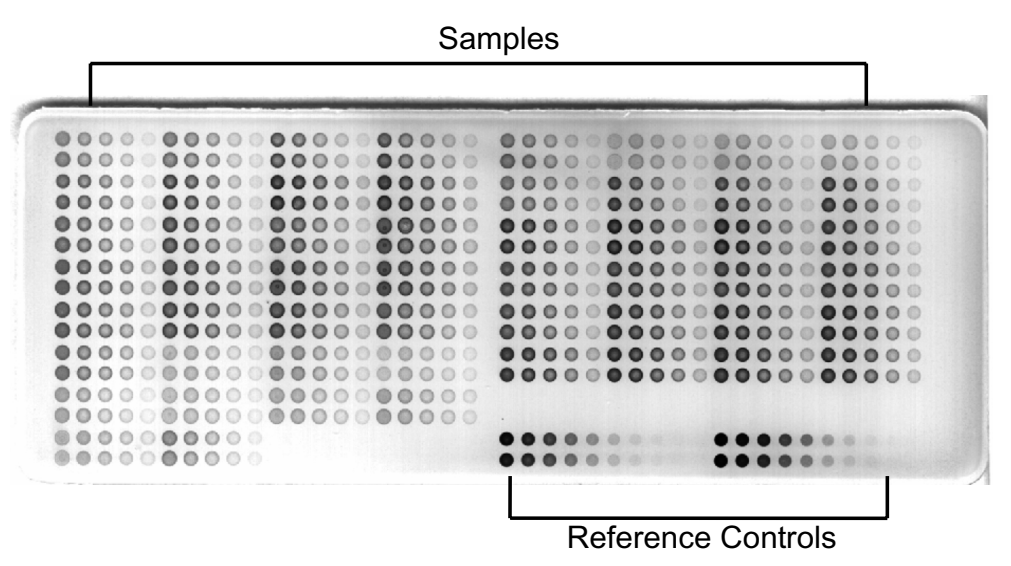

Fig. 2. Example of a Reverse phase protein microarray. Samples are spotted in a 5 point dilution curve to provide intensity values within the linear range. Reference positive control samples, in this case commercially available lysates, are also present in a 10 point dilution curve. This further enhances quantification and enables sample comparisons across slides.

samples in a dilution curve. In addition direct quantitative measurements are possible when comparing samples to reference spots that are printed on the same array (Fig. 2). Reproducibility is further enhanced due to the fact that direct tagging of the analyte is not required. All of these attributes combined result in a highly sensitive robust system [25,27].

\section{Disease understanding and treatment}

Many different causes can contribute to an altered protein activation pattern such as mutation, overexpression, or mutation of an interacting protein. Identification of activated or altered signaling networks that possibly correlate with disease progression or onset can be useful in the development of effective disease treatment and diagnosis. An ability to identify pivotal protein interactions or nodes within a signaling network that can be used as therapeutic targets or as biomarkers to monitor disease progression, drug responsiveness, or for diagnosis is necessary to develop more effective drug therapies and patient specific treatments. RPMAs have been used to gain a deeper understanding into various types of cancers, pathway characterizations, bacterial infection mechanisms, and immunological disorders $[5,12,13,20]$. These studies further exemplify the experimental versatility accommodated by the RPMA platform.

Many different experimental designs have been used to study various types of cancers such as studies examining colorectal, glioma, rhabdomyosarcoma, as well as studies characterizing the mechanism of anticancer agents. In the case of colorectal cancer, the experi- mental design involved the examination of differences in the activation levels of mitogen-activated protein kinase (MAPK) when comparing diseased stromal tissue to uninvolved tissue. Contrary to what had been traditionally thought it was found that the MAPK pathway in diseased tissue exhibited reduced activation when compared to the uninvolved tissue. This was established through the use of total protein antibodies as well as phospho-specific antibodies. This study was able to show that perhaps MAPK was not as ideal a potential drug target as previously thought [9]. RPMA have also been utilized to study the varying degrees of glioma, a tumor affecting the nervous system, and how their pathway alterations compare. This study was able to identify 12 proteins that showed higher expression levels in glioblastomas when compared to lower-grade tumors. Here the RPMA platform facilitated the identity of some proteins that may provide insight into tumor progression and aggressiveness in varying states of glioma [11]. In the case of rhabdomyosarcoma, $33 \%$ of children fail to respond to therapy regardless of disease stage or histological subtype, resulting in a need to identify functionally relevant networks that can be utilized for new treatment strategies. Through the use of RPMAs, a strong correlation between the activation of multiple interconnected Akt/mammalian target of rapamycin (mTOR) pathway components and poor overall survival was established. It was also found that insulin receptor substrate-1 (IRS-1) is subject to a negative feedback loop in response to Akt/mTOR activation. This feedback loop was found to be dysregulated in non-survivor samples. Here RPMAs were able to aid in identifying a potential pathway that can be monitored as a means to select patient treatment [18]. 
Lastly, RPMAs have been utilized to gain insight into the mechanism of the anticancer agent NSC-741909. Through monitoring of 77 protein biomarkers in cell lines derived from lung, colon, breast, ovary, and kidney cancers at different time points post treatment, it was found that sustained activation of c-jun N-terminal protein kinase (JNK) through inhibition of dephosphorylation was the main contributor to the apoptotic effects of NSC-741909. This was confirmed through the use of a JNK inhibitor and use of a JNK dominantnegative construct. Here the array displays its ability to process many different samples and endpoints with all the test conditions being consistent for each endpoint [25].

The RPMA was also utilized to generate experimental data regarding the timing of the p53-mouse double minute $2(\mathrm{Mdm} 2)$ feedback loop which has been well characterized and mathematically modeled, but the specifics of the timing events were largely lacking. This type of experimental design would generate a large number of samples from many different time points and would require a quantitative, high-throughput protein monitoring system. Here the RPMA allowed pathway characterization at the systems level through the usage of wild-type and knockout models to further elucidate the inner workings of this feedback loop [20].

The RPMA platform can also be applied to study the mechanism of infection by various microorganisms, in this case Salmonella, into the host cell. In this experiment, Salmonella lacking one or a few components thought to aid in infection and host cell re-programming were selected [12]. This approach allowed the signaling events that occur at various stages of infection to be monitored, in addition to an ability to monitor individual or joint contributions by various virulent factors depleted in the mutant bacterial populations. This study reveals the RPMA to provide the high throughput and small sample size for which it is touted. Utilization of this methodology can be further applied to studies of the infection process through examination of cultures of activated macrophages or other professional phagocytes exposed to various wild type and mutant bacteria to characterize the molecular determinants for resistance to phagocytosis in addition to other cellular aspects [12].

Lastly, RPMAs have also been utilized to study Tcell signaling. This study involved examination of $\mathrm{T}$ cell receptor signaling in Jurkat $\mathrm{T}$ cells. $\mathrm{T}$ cells were treated with CD3 or CD3 in combination with CD28. The vast array of phospho-endpoints examined revealed that CD28 did have an effect on the cells dependent on the exposure time. This platform enabled a large scale examination of the effects of treated cells, over the course of time with a broad variety of endpoints [5]. The above experiments demonstrate just a few of the experimental designs for which RPMAs can be utilized.

\subsection{Biomarker discovery and validation}

In addition to facilitating advancements in disease understanding, RPMAs have also aided in characterizing and identifying biomarkers. Events within the cell occur very rapidly, making it difficult to capture a real time image of these signaling events. The ability to obtain this imagery has significant applications in biomarker discovery. Monitoring the activation of a signaling pathway, in a patient's tumor, enables the determination of drug responsiveness when treated with an inhibitor targeting a node in a specific pathway, hence serving as a predictive biomarker. In addition, monitoring the activation status of a given protein preand post-treatment serves as a reliable indicator of the treatments effectiveness, acting as a pharmacodynamic biomarker. One such example, breast cancer falls into distinct molecular subtypes that vary in prognosis and drug response. The ability to do broad scale pathway monitoring utilizing the RPMA platform showed that the activation of certain pathways occurred in a subtype-specific manner [2]. These findings further pronounce the need for more fine tuned therapeutic treatments that target these differences.

RPMAs have also been diversely utilized for biomarker discovery and validation both in tissue sources and serum samples. One such example where the RPMA platform is able to out perform the current biomarker validation methods is seen in the case of pancreatic cancer biomarker validation. Currently, many potential biomarkers for pancreatic cancer exist, but their validation has been difficult due to cost and the sample size requirements when utilizing the conventional methods of an ELISA or Luminex assay. The biomarker CA19-9 is currently used in clinical practice to monitor the effects of cancer therapy and to detect recurrence in patients with pancreatic cancer. The results of this study showed that RPMAs can accurately detect CA19-9 levels. RPMA samples even showed greater sensitivity over ELISA samples when adjusted for potential loading differences. These results would suggest that RPMAs can be used reliably for rapid patient monitoring and validation of potential blood biomarkers [7]. 
The RPMA platform has also been utilized for biomarker discovery in the cases of colorectal cancer and non-alcoholic fatty liver disease [3,19]. In colorectal cancer very little is known about the molecular factors that determine the timing or progression toward metastasis. Insight into the signaling driving this transition may provide clues to enable early diagnosis and more effective treatment. This study examined the pathway signaling differences between patients with colorectal cancer that also experience metastasis and those who did not. Out of 81 endpoints that were examined only 9 were found to be significantly altered between the two groups. This approach enabled the identification of a few metastatasis-associated proteins that are statistically different between the two groups. These finding could result in the generation of more effective treatments, as well as potential biomarkers that can be used to determine patients with potential metastatic cancer [19]. In the case of nonalcoholic fatty liver disease (NAFLD), adipose tissue is being examined to determine the potential role it may play in disease development. It has been noted that there is a range in severity in NAFLD from simple steatosis (SS), which follows a more benign path, to nonalcoholic steatohepatitis (NASH). It was found that patients with NASH showed significant alterations in pathways relating to insulin resistance when compared to SS and obese only patients. This study demonstrated a new approach for potential tissue biomarkers found in adipose tissue that can accurately distinguish NASH from other types of NAFLD. After further clinical studies to validate these findings are performed, more effective treatments for the different variants of NAFLD through biomarker usage can be implemented [3].

While an ability to utilize serum biomarkers is ideal due to low invasiveness and availability, it has been more challenging to optimize the RPMA platform for this application. RPMA usage for serum and plasma samples has been limited to the detection of high abundance proteins. The objective of one experiment was to optimize the RPMA platform to detect a mid abundant blood protein called clusterin, a potential biomarker associated with carcinogenesis, tumorigenesis, and chemoresistance, which is present in the $\mu \mathrm{g} / \mathrm{mL}$ range. To optimize the detection of lower abundance proteins, three different slide types were examined to include nitrocellulose, epoxy coated glass slides, and MaxiSorp ${ }^{\mathrm{TM}}$ black polymer plastic slides. In addition, the samples were prepared in two different buffers, non-denaturing buffer (protein printing buffer) and a denaturing buffer (urea). The nitrocellulose pre- formed the best with urea buffer, but the conditions did not optimize attachment and higher background auto-fluorescence was noted. The most optimal platform was found to be epoxy coated slides with samples in protein printing buffer. These conditions facilitated low background, the lowest spot to spot variability, and showed fluorescent linearity with intensities corresponding to plasma serial dilutions. The benefit of epoxy coated slides relative to nitrocellulose is the reduced non-specific background that is seen in the epoxy slides. Epoxy coated slides also immobilize proteins covalently which provides a more diffuse distribution of proteins on the slide, where as the nitrocellulose immobilizes non-covalently (via hydrophobic interactions). While this study shows promise for detection of serum biomarkers, further optimization is required since most clinically relevant biomarkers are present in the $\mathrm{ng} / \mathrm{mL}$ range [1]. Another example of serum being utilized on the RPMA platform involves examination of individuals with IgA deficiency, one of the most common primary immunodificiencies found in Caucasians. The current method of using nephelometry requires higher sample quantities and low throughput, hence making it unsuitable for large scale screening and more costly then the RPMA platform. This study was able to show that when using epoxy coated slides spotted with serum samples, IgA levels as low as $1 \mu \mathrm{g} / \mathrm{mL}$ were detectable. The RPMA platform facilitated much higher throughput, in this case printing 2000 patient samples in triplicate, which would allow for rapid low sample size screening. It is also possible that if the serum had some of the higher abundant proteins like albumin and IgG removed that the sensitivity would increase even more [10].

\subsection{Applications for patient-tailored therapy}

The summation of the information gathered through a better disease understanding, in addition to identification of disease biomarkers, should result in more effective individualized treatment. An example of the need for individualized therapy can be seen when comparing primary ovarian samples with their metastatic counterparts. It was discovered that the metastatic tissue had developed a dramatically different protein signaling signature which appeared to be patient-specific. These findings just further point out the aberrant protein functioning, in particular kinase activity that can be at the root of many diseases. Given the vast array of molecular defects in ovarian cancer alone, it is inevitable that cancer agents will only suit a subset of 
patients during a specific stage of their disease. This is why the need to study several pathways simultaneously and develop a patient "fingerprint" is necessary for the most effective treatment [22]. It is hoped that this patient "fingerprint" can then aid in patient-specific network targeting. This would involve utilization of a patient's molecular profile map to assign an optimal regimen of therapies best suited to an individual's tumor. This profile or "fingerprint" can then be repeatedly monitored through biopsy or molecular imaging and the treatment regimen adjusted accordingly [17].

\subsection{Strengthening the platform}

While the RPMA exemplifies a robust platform, it also has areas for potential performance enhancement. One of the major limitations of the RPMA platform, like all other immunoassay platforms that require specific primary antibodies, is the availability of specific antibodies, in particular antibodies that detect post-translational modifications and activated protein states. However, fortunately, there now exists a large and growing selection of well-characterized, phosphospecific antibodies have become commercially available $[17,27]$. Another constant struggle has been proper selection of controls that will suit the largest array of studies. Two ways being examined to enhance the platform include use of a silicon membrane in place of nitrocellulose, as well as examination of other visualization platforms that allow for multiplexing and/or signaling enhancement.

Selection of proper controls to print in conjunction with each array that represent the largest array of samples is very difficult. Ideally in order to confidently compare across study sets there would be standardization of tissue handling and the development of a universal reference standard that would serve as a positive control. This universal standard would be deposited onto each array and allow compensation for variations in instrumentation, reagents, operators, and sample types thus enabling study comparisons across time. A good positive control reference standard should be renewable, provide reproducible results, provide accurate detection across a broad range of end points, and be stable over time. However it is very challenging to find a standard that can accommodate a wide range of tissue types and study sets. In some cases a cocktail of phosphorylated peptides specific for antibodies commonly used is beneficial. In others a commercial lysate or several lysates is more beneficial by providing broader coverage of potential end points [22,27].
Another approach is the use of an internal control of a selected protein present across all samples, for example $\beta$-actin. Here the samples were detected through probing with $\mathrm{Cy} 3$-Streptavidin and $\beta$-actin was detected through a Cy5-Streptavidin label. Each Cy3 background subtracted intensity value was then normalized to the levels of $\beta$-actin [5]. Another example of utilizing an internal control is seen when using serum samples. Here an internal control of $\mathrm{IgG}$ or albumin can be chosen. In a study examining both cancerous and benign samples, similar levels of albumin were noted making it a suitable protein for normalization. The normalization of samples to albumin resulted in an increase in sensitivity from $73 \%$ to $90 \%$ when compared to non-normalized samples [7].

While nitrocellulose has provided a conducive substratum for RPMAs due to its high protein binding abilities, it does have a higher propensity toward auto-fluorescence. Recently, investigators have developed and evaluated silicon as an alternative printing surface due to it low auto-fluorescence. However, in order to optimize the silicon for use, it requires physciochemical modifications. There are two different methods that have been examined to optimize this substrate to result in a membrane that can compete with or potentially out perform its nitrocellulose counterpart. One method involves combining usage of titrate reactive ion etching with chemical treatment. Out of various etching times and chemical treatment combinations that were explored, etching for $20 \mathrm{~min}$ utes in combination with mercaptopropyltrimethoxsilane (MPTMS) treatment resulted in a protein-binding capacity that exceeded the other combinations in addition to the nitrocellulose [14]. However, in a later study, 3-aminopropyltriethoxysaline (ATPES) etched for 180 minutes showed the highest signal after being normalized to the background, even in comparison to nitrocellulose. This may appear contrary to the previous study. However previously, spot morphology was not taken into account or which combination resulted in the cleanest normalized data. Overall, these experiments show that the system could be further optimized by experimentation with different solvent compositions to optimize signaling [15]. An alternative method of silicon utilization employs the use of p-type silicon that is anodized in a hydrofluoric acid/DMF solution. This results in 3-D wafers that are then adhered to a plastic slide, with samples being printed using a non-contact mode. This design results in a surface enlargement coupled with a low surface wetting and high droplet contact angle resulting in small spot size, improved re- 
action kinetics, and high density arraying. The performance of this platform was validated through detection of the breast cancer biomarker cyclin E. This protein was detected in several cell lines, human tissue lysates, and samples with spiked in levels of cyclin E. Samples were spotted with approximately $100 \mathrm{pL}$ droplet size resulting in spots between $50-70 \mu \mathrm{m}$ in size enabling printing densities of up to $14,000 \mathrm{spots} / \mathrm{cm}^{2}$. Ultimately, this approach was shown to yield good spot quality and showed no difficulty in detection of varying levels of cyclin $E$ between samples. These findings would indicate that the current sensitivity of the assay would be able to meet the demands of the RPMA platform [21].

It is important when selecting a visualization method that a strong, clean signal is obtained. When using a chromogenic detection method, detection is limited to one protein target per slide and requires an amplification step such as biotinyltyramide. Whereas a fluorophore based detection system, such as quantum dots, could offer a more versatile system with the ability to multiplex and observe multiple protein targets on a single array. Among fluorescent reporters, organic fluorophores are prone to bleaching with prolonged exposure and tend to have a broad emission and narrow excitation spectra, hence limiting their usefulness in multiplexing. However quantum dots for example, display a narrow emission band size, broad excitation band size, and a large extinction coefficient resulting in a more robust fluorescent signal. Quantum dots are semiconducting nanocrystals ranging is size from 1 to $10 \mathrm{~nm}$ that are able to generate a bright fluorescent signal. This signal is generated through the reporter being linked to streptavidin and recognizing the biotinylated secondary antibody, rather then detection by an enzyme such as horseradish peroxidase as in the case of the colormetic system. The use of a quantum dot detection system holds promise for multiplexing due to its ability to resist photobleaching while having a wide excitation and narrow emission spectra [6,15]. A further iteration of RPMA detection methods utilize enzymatic amplification with near-IR dyes, and while not providing the high degree of multiplexing capabilities of quantum dots, these dyes have provided an extremely reliable means of stable and sensitive detection for RPMA. Utilization of these dyes enables a much higher density array that eliminates the need for printing multiple separate serial dilutions of the same sample due to the much larger within-spot dynamic range (approx 300-500 times) compared to the 2-fold range of colorimetric assays [4].

\subsection{A view to the near future}

The largest constraint on any analytical process such as the RPMA is how it translates into commercial implementation and routine use. The RPMA has the ability to batch process varying patient samples while screening for a single protein. Because it can generate multiplexed and quantitative measurements for entire protein signaling networks, it has unparalleled ability for drug target pathway mapping from tiny biopsy specimens. In theory this would allow for large volume patient-tailored lab testing. The platform is able to accommodate almost any experimental design from disease study, to time courses, and biomarker validation and screening. While genomic and proteomic efforts are yielding a large collection of candidate molecules potentially relevant to disease diagnostics or therapeutics, the RPMA is well equipped to expand upon the initial analysis and provide answers as to the overall applicability of the biomarkers through quantitative measurements across many samples/patients at once. The platform can provide data beyond the analysis of whether or not the candidate molecule is present or altered during the disease process, but also generate data on if the alterations transcend patient history and results in an altered molecular phenotype in a majority of patients [16]. The RPMA is proving itself to be a tremendously effective tool through which we can define diseases by functional pathway mapping that could provide more effective patient specific treatment.

\section{References}

[1] A. Aguilar-Mahecha, C. Cantin, M. O'Connor-McCourt, A. Nantel and M. Basik, Development of reverse phase protein microarrays for the validation of clusterin, a mid-abundant blood biomarker, Proteome Sci 7 (2009).

[2] Z. Boyd, Q. Wu, C. O'Brian, J. Spoerke, H. Savage, P. Fielder, L. Amler, Y. Yan and M. Lackner, Proteomic analysis of breast cancer molecular subtypes and biomarkers of response to targeted kinase inhibitors using reverse-phase protein microarrays, Mol Cancer Ther 7 (2008), 3695-3706.

[3] V. Calvert, R. Collantes, H. Elariny, A. Afendy, A. Baranova, M. Mendoza, Z. Goodman, L. Liotta, E. Petricoin and Z. Younossi, A systems biology approach to the pathogenesis of obesity-related nonalcoholic fatty liver disease using reverse phase protein microarrays for multiplexed cell signaling analysis, Hepatology 46 (2007), 166-172.

[4] V. Calvert, Y. Tang, V. Boveia, J. Wulfkuhle, A. SchutzGeschwender, M. Olive, L. Liotta and E. Petricoin, Development of multiplexed protein profiling and detection using near infrared detection of reverse-phase protein microarrays, Clinical Proteomics 1 (2004), 81-90. 
[5] S. Chan, J. Ermann, L. Su, C. Garrison Fathman and P. Utz, Protein microarrays for multiplex analysis of signal transduction pathways, Nat Med 10 (2004), 1390-1396.

[6] D. Geho, J. Killian, A. Nand, J. Pastor, P. Gurnani and K. Rosenblatt, Fluorescence-based analysis of cellular protein lysate arrays using quantum dots, Methods Mol Biol 374 (2007), 229-237.

[7] T. Grote, D. Siwak, H. Fritsche, C. Joy, G. Mills, D. Simeone, D. Whitcomb and C. Logsdon, Validation of reverse phase protein array for practical screening of potential biomarkers in serum and plasma: accurate detection of CA19-9 levels in pancreatic cancer, Proteomics 8 (2008), 3051-3060.

[8] R. Grubb, J. Deng, P. Pinto, J. Mohler, A. Chinnaiyan, M. Rubin, W. Linehan, L. Liotta, E. Petricoin and J. Wulfkuhle, Pathway biomarker profiling of localized and metastatic human prostate cancer reveal metastatic and prognostic signatures, $J$ Proteome Res 8 (2009), 3044-3054.

[9] C. Gulmann, K. Sheehan, R. Conroy, J. Wulfkuhle, V. Espina, M. Mullarkey, E. Kay, L. Liotta and E. Petricoin, Quantitative cell signaling analysis reveals down-regulation of MAPK pathway activation in colorectal cancer, J Pathol 218 (2009), 514-519.

[10] M. Janzi, J. Ödling, Q. Pan-Hammarström, M. Sundberg, J. Lundeberg, M. Uhlén, L. Hammarström and P. Nilsson, Serum microarrays for large scale screening of protein levels, $\mathrm{Mol}$ Cell Proteomics 4 (2005), 1942-1947.

[11] R. Jiang, C. Mircean, I. Shmulevich, D. Cogdell, Y. Jia, I. Tabus, K. Aldape, R. Sawaya, J. Bruner, G. Fuller and W. Zhang, Pathway alterations during glioma progression revealed by reverse phase protein lysate array, Proteomics 6 (2006), 2964-2971.

[12] C. Molero, I. Rodríguez-Escudero, A. Alemán, R. Rotger, M. Molina and V. Cid, Addressing the effects of Salmonella internalization in host cell signaling on a reverse-phase protein array, Proteomics 9 (2009), 1-14.

[13] E. Neeley, S. Kornblau, K. Coombes and K. Baggerly, Variable slope normalization of reverse phase protein arrays, Bioinformatics 25 (2009), 1384-1389.

[14] A. Nijdam, M. Cheng, D. Geho, R. Fedele, P. Herrmann, K. Killian, V. Espina, E. Petricoin, L. Liotta and M. Ferrari, Physicochemically modified silicon as a substrate for protein microarrays, Biomaterials 28 (2007), 550-558.

[15] A. Nijdam, M. Zianni, E. Herderick, M. Cheng, J. Prosperi, F. Robertson, E. Petricoin, L. Liotta and M. Ferrari, Application of physicochemically modified silicon substrates as reversephase protein microarrays, J Proteome Res 8 (2008), 12471254.

[16] C. Paweletz, L. Charboneau, V. Bichsel, N. Simone, T. Chen, J. Gillespie, M. Emmert-Buck, M. Roth, E. Petricoin and L. Liotta, Reverse phase protein microarrays which capture disease progression show activation of pro-survival pathways at the cancer invasion front, Oncogene 20 (2001), 1981-1989.

[17] E. Petricoin, V. Bichsel, V. Calvert, V. Espina, M. Winters, L. Young, C. Belluco, B. Trock, M. Lippman, D. Fishman, D. Sgroi, P. Munson, L. Esserman and L. Liotta, Mapping molecular networks using proteomics: a vision for patient- tailored combination therapy, J Clin Oncol 23 (2005), 36143621.

[18] E. Petricoin, V. Espina, R. Araujo, B. Midura, C. Yeung, X. Wan, G. Eichler, D. Johann, S. Qualman, M. Tsokos, K. Krishnan, L. Helman and L. Liotta, Phosphoprotein pathway mapping: Akt/mammalian target of rapamycin activation is negatively associated with childhood rhabdomyosarcoma survival, Cancer Res 67 (2007), 3431-3440.

[19] M. Pierobon, V. Calvert, C. Belluco, E. Garaci, J. Deng, M. Lise, D. Nitti, E. Mammano, F. De Marchi, L. Liotta and E. Petricoin, Multiplexed cell signaling analysis of metastatic and nonmetastatic colorectal cancer reveals COX2-EGFR signaling activation as a potential prognostic pathway biomarker, J Proteome Res 8 (2009), 1247-1254.

[20] S. Ramalingam, P. Honkanen, L. Young, T. Shimura, J. Austin, P. Steeg and S. Nishizuka, Quantitative assessment of the p53Mdm2 feedback loop using protein lysate microarrays, Cancer Res 67 (2007), 6247-6252.

[21] A. Ressine, I. Corin, K. Järas, G. Guanti, C. Simone, G. Marko-Varga and T. Laurell, Porous silicon surfaces - a candidate substrate for reverse protein arrays in cancer biomarker detection, Electrophoresis 28 (2007), 4407-4415.

[22] K. Sheehan, V. Calvert, E. Kay, Y. Lu, D. Fishman, V. Espina, J. Aquino, R. Speer, R. Araujo, G. Mills, L. Liotta, E. Petricoin and J. Wulfkuhle, Use of reverse phase protein microarrays and reference standard development for molecular network analysis of metastatic ovarian carcinoma, Mol Cell Proteomics 4 (2005), 346-355.

[23] R. Tibes, Y. Qui, Y. Lu, B. Hennessy, M. Andreeff, G. Mills and S. Kornblau, Reverse phase protein array: Validation of a novel proteomic technology and utility for analysis of primary leukemia specimens and hematopoietc stem cells, Mol Cancer Ther 5 (2006), 2512-2521.

[24] A. Vanmeter, A. Rodriguez, E. Bowman, J. Jen, C. Harris, J. Deng, V. Calvert, A. Silvestri, C. Fredolini, V. Chandhoke, E. Petricoin, L. Liotta and V. Espina, Laser capture microdissection and protein microarray analysis of human non-small cell lung cancer, Mol Cell Proteomics 7 (2008), 1902-1924.

[25] X. Wei, W. Guo, S. Wu, L. Wang, Y. Lu, B. Xu, J. Lui and B. Fang, Inhibiting JNK dephosphorylation and induction of apoptosis by novel anticancer agent NSC-741909 in cancer cells, J Biol Chem 284 (2009), 16948-16955.

[26] S. Wong, C. Chan, B. Ma, M. Lam, G. Choi, T. Au, A. Chan and A. Chan, Advanced proteomic technologies for cancer biomarker discovery, Expert Rev Proteomics 6 (2009), 123134.

[27] J. Wulfkuhle, K. Edminston, L. Liotta and E. Petricoin, Technology Insight: pharmacoproteomics for cancer-promises of patient-tailored medicine using protein microarrays, Nat Clin Pract Oncol 3 (2006), 256-268.

[28] J. Wulfkuhle, R. Speer, M. Pierobon, J. Laird, V. Espina, J. Deng, E. Mammano, S. Yang, S. Swain, D. Nitti, L. Esserman, C. Belluco, L. Liotta and E. Petricoin, Multiplexed cell signaling analysis of human breast cancer applications for personalized therapy, J Proteome Res 7 (2008), 1508-1517. 


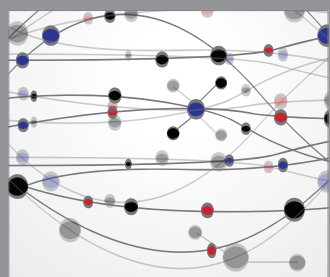

The Scientific World Journal
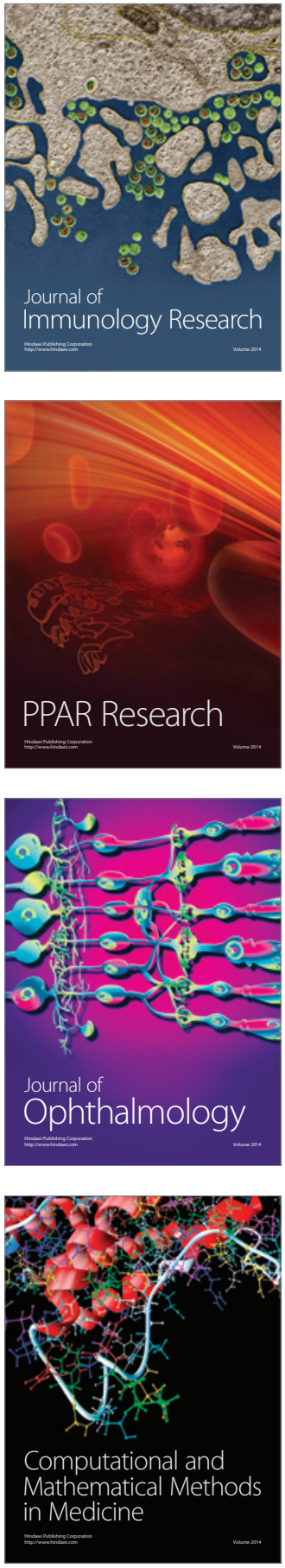

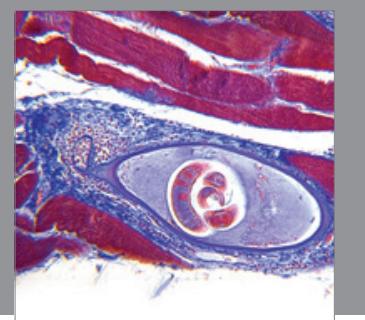

Gastroenterology

Research and Practice
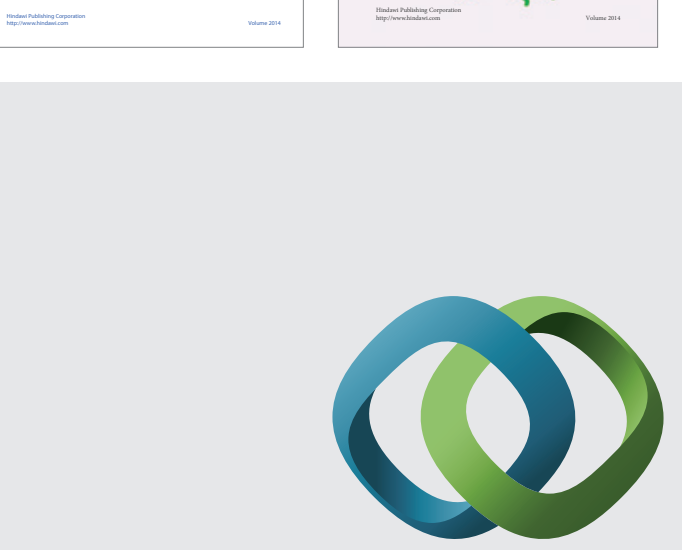

\section{Hindawi}

Submit your manuscripts at

http://www.hindawi.com
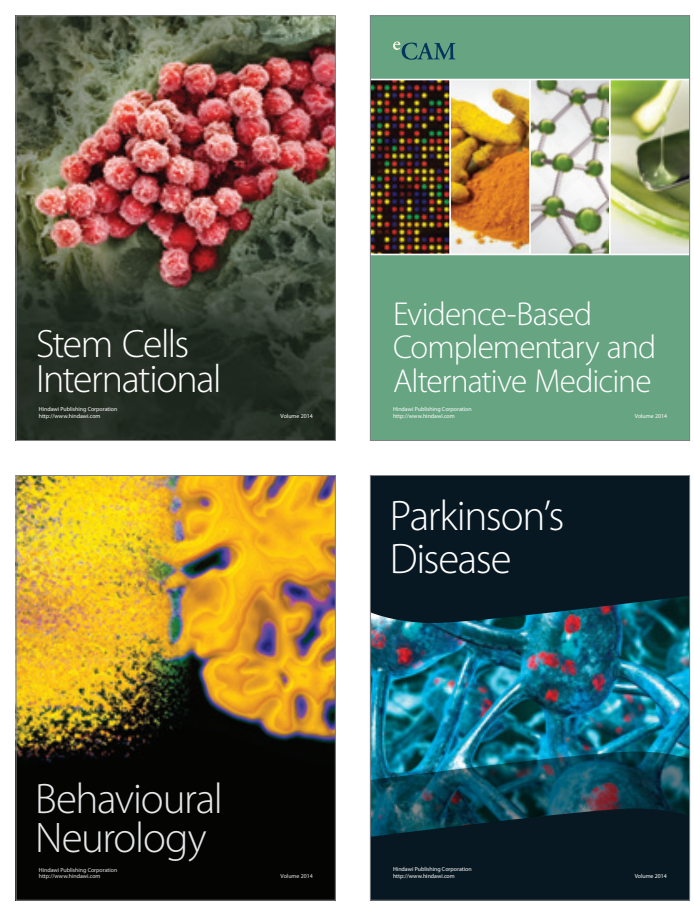

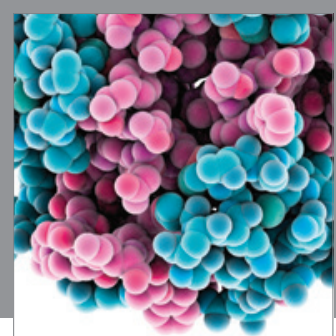

Journal of
Diabetes Research

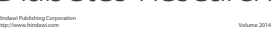

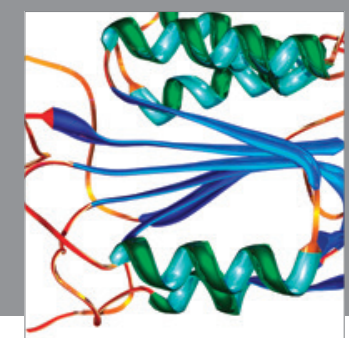

Disease Markers
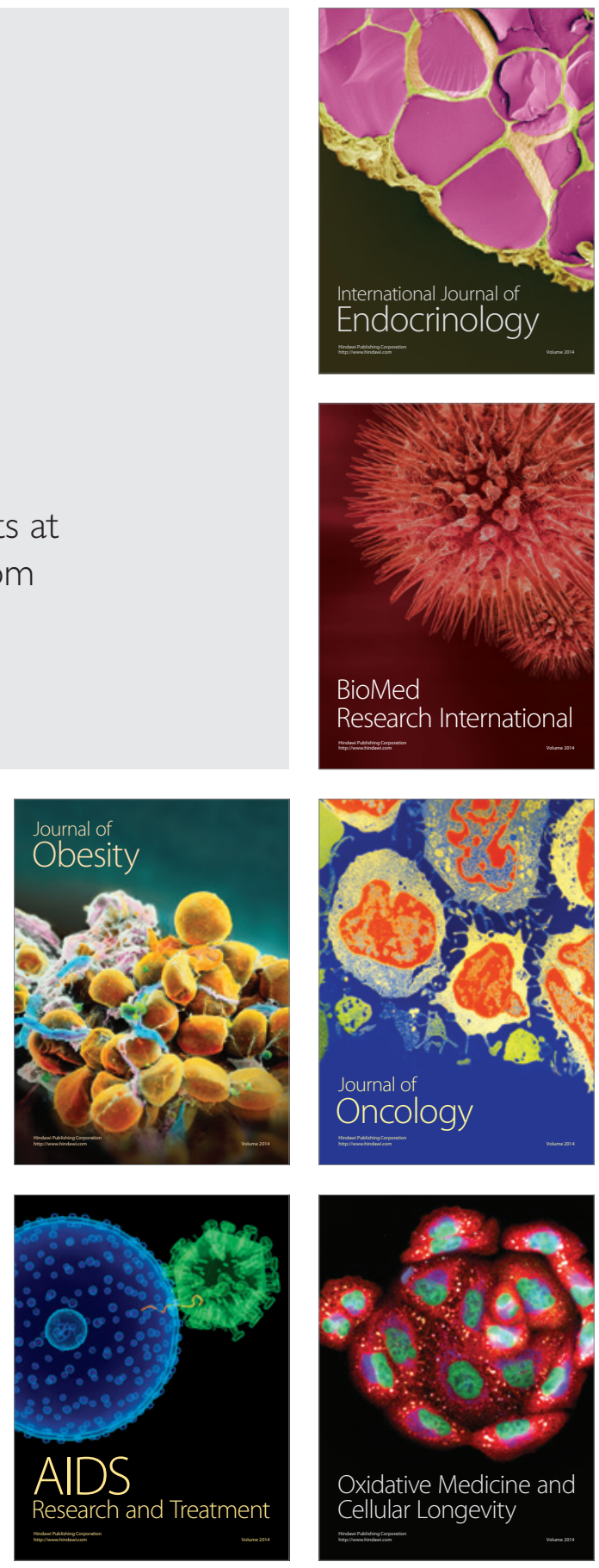